\title{
Effects of aluminum and manganese impurity concentrations on optoelectronic properties of thin films of Tin Sulfide (SnS) using CBD method.
}

\author{
J. C. Osuwa ${ }^{1}$ and J. Ugochukwu ${ }^{2}$ \\ Department of Physics, Michael Okpara University of Agriculture, Umudike PMB 7267, Abia State, Nigeria
}

\begin{abstract}
Thin films of Tin Sulfide (SnS) with varying concentrations of aluminum and manganese impurities (0.01-0.04M) were grown on glass substrates at room temperature in a chemical bath containing Tin II chloride, sodium thiosuphate, sodium ethylene diaminetetra acetate EDTA, ammonia and sodium tri-citrate. The optoelectronic properties of all the films were analyzed using a spectrophotometer and four point probe. The results show generally low transmittance and reflectance with the highest increase of $3 \%$ in transmittance occurring for 0.01M of aluminum and 0.02M of manganese impurities. The reflectance increased to $9 \%$ for $0.04 \mathrm{M}$ of aluminum and $15 \%$ for $0.02 \mathrm{M}$ of manganese impurities while $0.01 \mathrm{M}$ of both impurities lowered the reflectance. The absorbance for all impurity concentrations converged from a high value of 1.0 in the UV region and decreased marginally to a minimum of 0.82 for $0.02 \mathrm{M}$ of manganese impurity in the visible region. Absorption coefficients increased with impurity concentration for both impurities. Also, the direct energy band gap increased with impurity concentration from $1.50 \mathrm{eV}$ for as grown thin film to (1.70-1.90 eV) for various concentrations of both impurities. Changes with impurity concentrations in the values of refractive index, optical conductivity and dielectric constant etc. are also reported here. Results of the four point probe revealed increase in electrical resistivity for both manganese and aluminum impurities, with a maximum at 0.03 molar concentration of aluminum.
\end{abstract}

Keywords: Tin Sulfide, optoelectronic properties, aluminum and manganese impurity concentrations.

\section{Introduction}

Tin sulfide $(\mathrm{SnS})$ belongs to IV-VI compound semiconductor materials with p-type electrical conductivity [1]. The constituent elements of tin and sulfur are nontoxic and abundant in nature leading to development of devices that are environmentally safe and have public acceptability. A high conversion efficiency of approximately $25 \%$ is attainable in photovoltaic devices using $\mathrm{SnS}$ thin films [1].The disulphide phase thin films of tin $\left(\mathrm{SnS}_{2}\right)$ are useful in current controlled devices, switching devices and photo-conducting cells [1-4].

Variations in the properties and diversity in the applications of thin films can generally be achieved through the use of different precursor concentrations, deposition time and temperature, deposition method, $\mathrm{pH}$ of solution and introduction of impurity ions [5-12]. The focus in this study is to obtain the effects of aluminum and manganese impurities on the optoelectronic properties of tin sulfide $(\mathrm{SnS})$ thin films deposited by $\mathrm{CBD}$ method at room temperature.

\subsection{Materials and Methods}

\section{Experimental Details}

Tin sulfide thin films were deposited using chemical bath deposition method. Substrates were degreased in tri-oxonitrate $(\mathrm{V})$ acid for 48 hours, rinsed with distilled water and dried in air. All solutions were prepared in de-ionized water. In the deposition process, $10 \mathrm{ml}$ of $0.1 \mathrm{M}$ Tin (II) chloride $\left(\mathrm{SnCl}_{2}\right)$ was complexed with $5 \mathrm{ml}$ of $0.1 \mathrm{M}$ Sodium EDTA agent and $5 \mathrm{ml}$ of $0.66 \mathrm{M}$ Sodium tri-citrate to reduce coagulation of the formed precipitate. To this, $10 \mathrm{ml}$ of $0.3 \mathrm{M}$ of Sodium thiosulphate $\mathrm{Na}_{2} \mathrm{~S}_{2} \mathrm{O}_{3} .5 \mathrm{H}_{2} \mathrm{O}$ was added and the reaction mixture was stirred. The $\mathrm{pH}$ of the chemical bath was maintained at 10.0 by addition of $2 \mathrm{ml}$ of ammonia. The resulting solution was stirred thoroughly and clean glass substrate was vertically immersed in the solution bath at room temperature for 24 hours. After deposition, the substrate was taken out of the reaction bath, washed with distilled water and dried in air. Other thin film samples were prepared with varying concentrations $(0.01-0.04 \mathrm{M})$ of aluminum and manganese impurity ions derived from aluminum chloride and manganese sulphate respectively. Equations (1) below show the chemical reactions.

$$
\begin{aligned}
& \mathrm{SnCl}_{2} \cdot 2 \mathrm{H}_{2} \mathrm{O}+[\text { EDTA }] \rightarrow[\mathrm{Sn}(\text { EDTA })]^{2+}+2 \mathrm{Cl}+2 \mathrm{H}_{2} \mathrm{O} \\
& {[\mathrm{Sn}(\text { EDTA })]^{2+} \rightarrow \mathrm{Sn}^{2+}+\text { EDTA }}
\end{aligned}
$$




$$
\begin{aligned}
& \mathrm{Na}_{2} \mathrm{~S}_{2} \mathrm{O}_{3} .5 \mathrm{H}_{2} \mathrm{O} \rightarrow \mathrm{Na}_{2} \mathrm{O}_{3}+5 \mathrm{H}_{2} \mathrm{O}+\mathrm{S}^{2-} \\
& \mathrm{Sn}^{2+}+\mathrm{S}^{2-} \rightarrow \mathrm{SnS}
\end{aligned}
$$

\subsection{Characterization of $\mathrm{SnS}$ thin films}

The transmittance and reflectance of both doped and un-doped $\mathrm{SnS}$ thin films were measured within the Vis-UV-IR spectral region using a spectrophotometer. The reference and film coated glass slides were mounted on a rotating holder at the reference and sample compartments of the spectrophotometer. Other optical, solid-state and electrical properties were obtained theoretically from the spectral data. Graphical representations of variations in the properties are shown in the sections that follow.

\section{Results and discussions}

Figures 1 (a) and (b) show that the transmittance of SnS thin films are generally low but increases with impurity concentration with highest value of about $3 \%$ occurring for $0.01 \mathrm{M}$ aluminum and $0.02 \mathrm{M}$ manganese impurities. Figures 2 (a) and (b) show lowest reflectance values for $0.01 \mathrm{M}$ of both impurity ions while the highest values of $9 \%$ and $15 \%$ respectively, occur for $0.04 \mathrm{M}$ of aluminum and $0.02 \mathrm{M}$ of manganese impurities. High absorbance values between 0.82-1.0 are obtained from the absorbance spectra of fig.2 (c) and (d). The absorption coefficients of SnS thin films in fig. 2 (e) and (f) show steady slight increase with increase in both impurity

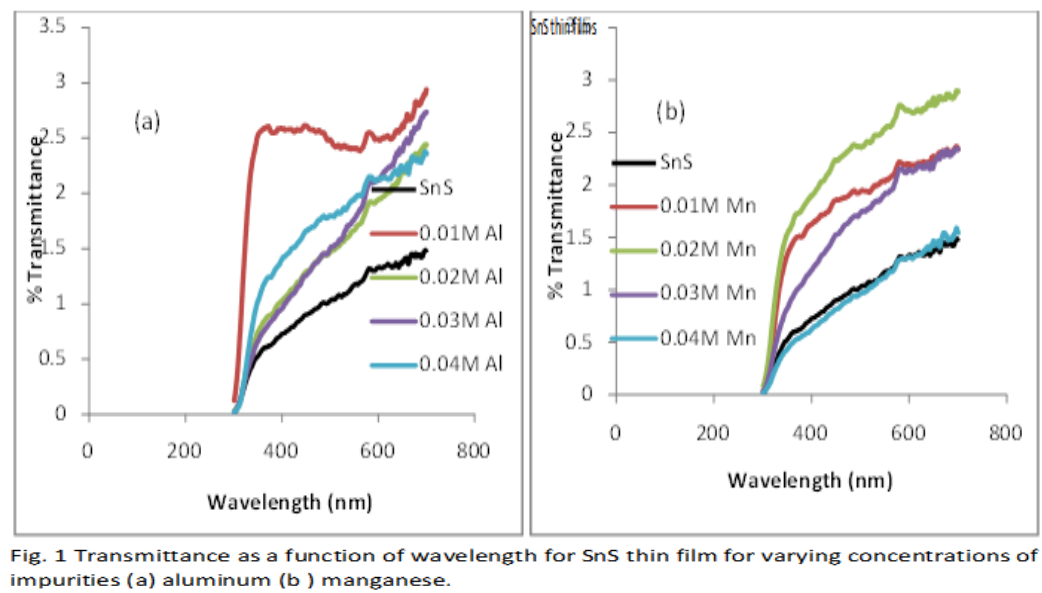

concentrations as well as slight decrease with decrease in photon energy.

In figures 3 (a) and (b) the energy band gap for both aluminum and manganese impurities increases with impurity concentration from $1.50 \mathrm{eV}$ for as grown thin film to $1.90 \mathrm{eV}$ for 0.04 impurity ions. The extinction coefficients fig. 3 (c) and (d) increase with impurity concentrations and decrease with increase in photon energy. Variations in refractive index from 1.0 to 1.18 are shown in fig. 3 (e) and (f). Changes in both real and imaginary constants for different impurity concentrations are shown in fig.4 (a-d) while the optical conductivity in fig. 4 (e) and (d) show steady slight increase with increase in both impurity concentrations and fairly constant values for all photon energy.

Figure 5 (a) shows decrease in film thickness with increase in both impurity concentrations while the resistivity of the $\mathrm{SnS}$ thin films increases with increase in impurity concentrations as shown in fig. 5 (b) with a maximum at $0.03 \mathrm{M}$ for aluminum impurity. 

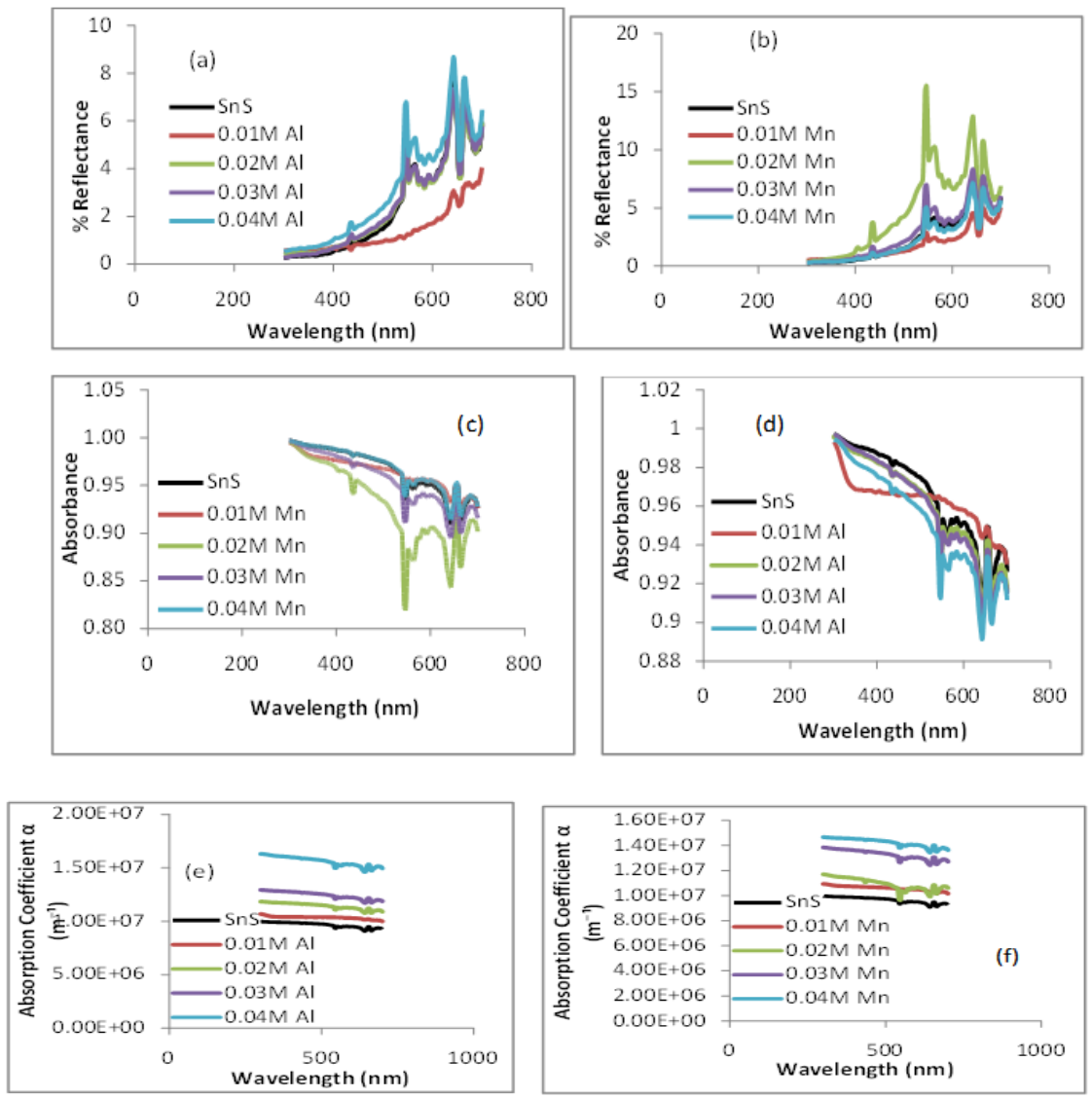

Fig. 2 Reflectance, absorbance and absorption coefficient as functions of wavelength for SnS thin films for varying impurity concentrations (a), (d) and (e) aluminum (b), (c) and (f) manganese.
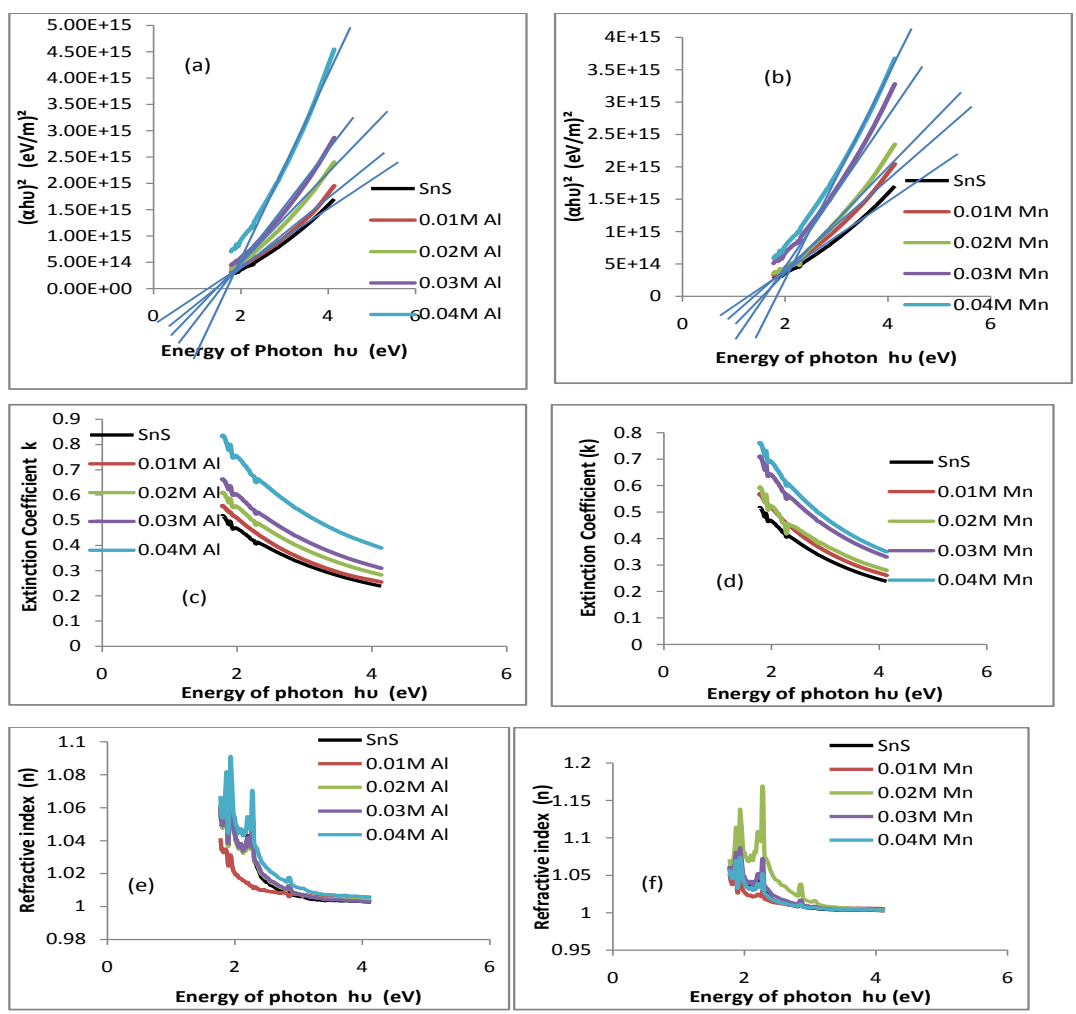

Fig. 3: Energy band gap, extinction coefficient and refractive index as functions of photon energy for Sns thin films for varying concentrations of impurities (a), (c) and (e) aluminum (b), (d) and (f) manganese. 

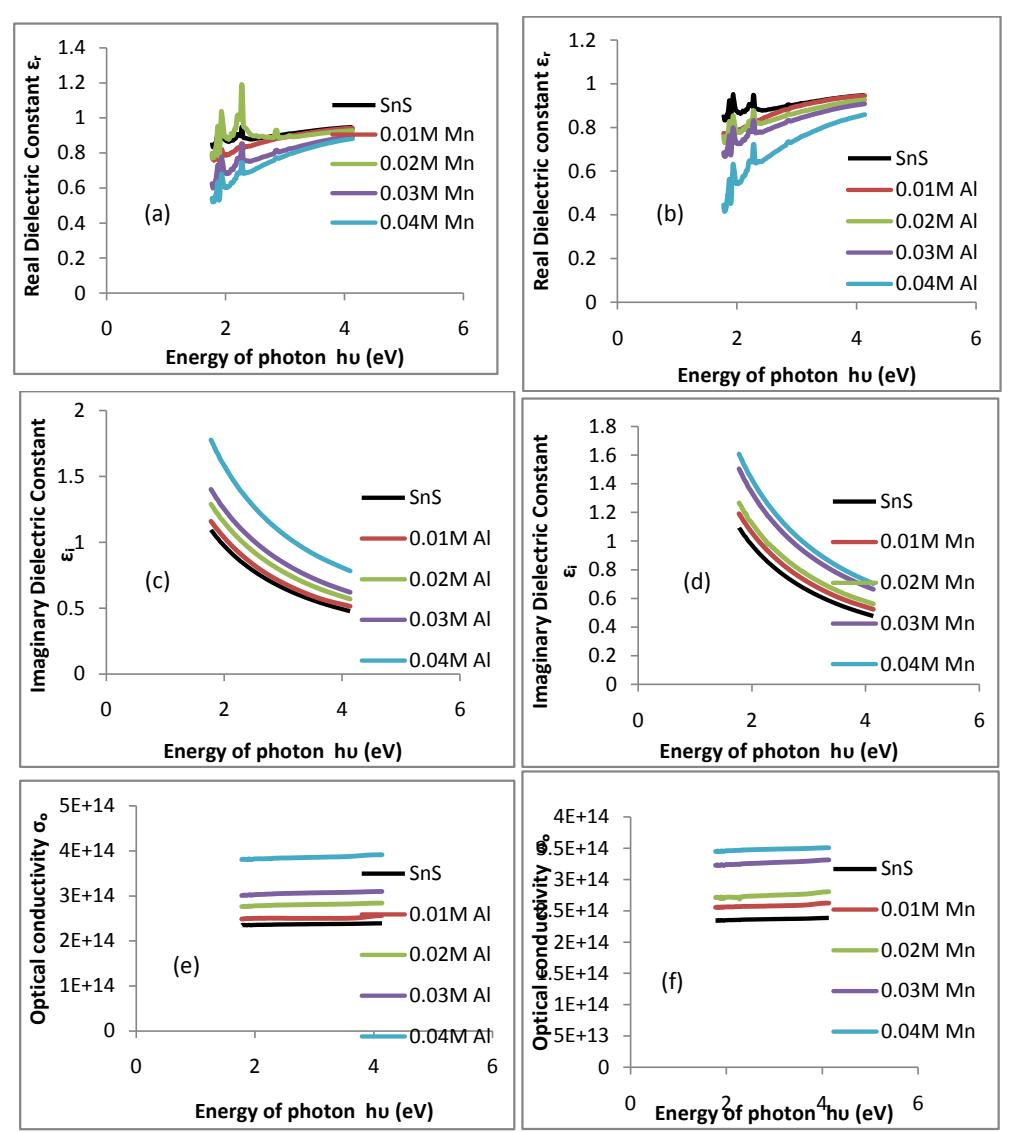

Fig. 4:Real and imaginary dielectric constants and optical conductivity as functions of photon energy for SnS thin films for varying concentrations of impurities (b), (c) and (e) aluminum (a) (d) and (f) manganese
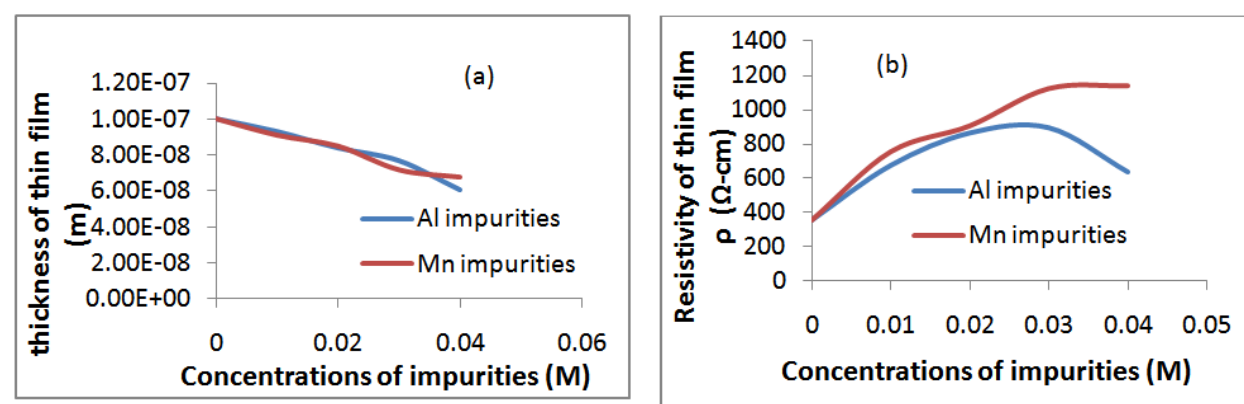

Fig. 5: (a) Thickness and (b) electrical resistivity of SnS thin films for varying concentrations of aluminum and manganese impurities.

\section{Conclusion}

Effects of aluminum and manganese impurities on the optoelectronic properties of CBD deposited tin sulfide $(\mathrm{SnS})$ thin films have been successfully investigated. Distinctive variations in the film properties for different impurity concentrations were obtained as presented in this paper. The results are characterized by high absorption coefficients of $\left(1.0 \times 10^{7}-1.6 \times 10^{7} \mathrm{~m}^{-1}\right)$ and consequent high absorbance of $(0.82-1.0)$ in conformity with relatively high conversion efficiency of $25 \%$ obtainable in photovoltaic applications of SnS thin films. The results also show among others, increase in the energy band gap form $1.50 \mathrm{eV}$ to $1.90 \mathrm{eV}$ as well as increase in resistivity of the thin films with increase in impurity concentrations. 


\section{References}

[1] Pathan, H.M, Lokhande, C. D, (2004) Deposition of metal chalcogenide thin films by successive ionic layer and adsorption and reaction (SILAR) method. Bull. Mater. Sci. Vol. 27 No. 2 pp. 85-111

[2] Patil, S. G and Tredgold, R, H (1971) Electrical and photo-conductive properties of SnS 2 crystals. J. Phys. D: Appl. Phys. 4 718.

[3] Said, G and Lee P. A (1973) Electrical conduction-mechanisms in tin disulphide. Phys. Status Solidi (a) 1599.

[4] Sankapal, B. R, Mane, R. S and Lokhande, C. D (2000c) Successive ionic layer adsorption and Reaction (SILAR) for deposition of large area $\left(\sim 10 \mathrm{~cm}^{2}\right)$ tin disulphide $\left(\mathrm{SnS}_{2}\right)$ thin films. Mater. Bull.35 2027.

[5] Osuwa, J. C and Onyejiuwa, G. I (2013) structural and Electrical properties of annealed Nickel Oxide (NiO) thin films prepare d by chemical bath deposition. Journal of Ovonic Research. 9 (1): 9-15

[6] Annuar, K, Zulkarnaain, Z, saravanan, N, Zuriyatine, A and Sharin, R (2004) Preparation and studies ofNickel Sulphide Thin Films in the presence of Sodium Tartate as a complexing Agent. Material Science 10 (2): 4-7

[7] Ezenwa, I, A (2013)Effects of deposition time on the absorbance on chemical bath deposited CuS thin films Research Journal of engineering sciences. 2(1): 1-4.

[8] Huda Abdullah, Norhabibi Saadah and Sahbuddin Shaari (2012) Effect of deposition time on ZnS thin film properties by chemical bath deposition (CBD) technique. World Applied Sciences Journal. 19 (8): 1087-1091

[9] Ezeokoye, B. A and Okeke C. E (2006) Optical properties in PbHgS Tenary thin films deposited by solution growth method. Pacific Journal of Science and Technology. 7 (2): 100-113.

[10] Sartale, S. D and Lokhande, C. D (2001) Preparation and characterization of Nickel Sulphide Thin Films using Successive Ionic Layer Adsorption and Reaction (SILAR). Material Chem. and Phys. 72 (8): 100-104

[11] Ezema, F. I, Ekwealor, A. B. C and Osuji, R. U (2006) Effect of thermal Annealing on the band gaps and optical properties of chemical bath deposited ZnSc thin films. Turkish Journal of Physics. 30, 157-168.

[12] Panta, G. P, Subedi D. P (2012) Electrical characterization of Aluminum (Al) Thin Films Measured By Using Four-Point Probe method. Kathmandu University journal of science, engineering and technology. Vol. 8, pp 31-36 